\title{
Feasibility and Outcomes of Cytoreductive Surgery \& HIPEC for Peritoneal Surface Malignancies in Low and Middle Income Countries: A Single centre Experience of 232 Cases
}

Suryanarayana Deo ( $\nabla$ svsdeo@yahoo.co.in )

All India Institute of Medical Sciences

Mukurdipi Ray

All India Institute of Medical Sciences

Babul Bansal

All India Institute of Medical Sciences

Sandeep Bhoriwal

All India Institute of Medical Sciences

\section{Sushma Bhatnagar}

All India Institute of Medical Sciences

Rakesh Garg

All India Institute of Medical Sciences

Nishkarsh Gupta

All India Institute of Medical Sciences

Atul Sharma

All India Institute of Medical Sciences

Lalit Kumar

All India Institute of Medical Sciences

Sanjay Thulkar

All India Institute of Medical Sciences

Ekta Dhamija

All India Institute of Medical Sciences

Sandeep Mathur

All India Institute of Medical Sciences

Prasenjit Das

All India Institute of Medical Sciences

Research Article 
Keywords: Peritoneal Surface Malignancy, Cytoreductive surgery, HIPEC, LMIC, Tertiary care centre Posted Date: March 17th, 2021

DOI: https://doi.org/10.21203/rs.3.rs-314520/v1

License: (c) (1) This work is licensed under a Creative Commons Attribution 4.0 International License. Read Full License 


\section{Abstract}

\section{Background:}

Cytoreductive surgery (CRS) and hyperthermic intraperitoneal chemotherapy (HIPEC) has recently emerged as a viable management option for peritoneal surface malignancy (PSM). CRS and HIPEC is a complex, multidisciplinary and resource intensive surgical option with a steep learning curve and significant morbidity and mortality. Most of experience is limited to few dedicated high volume centres from developed countries. We present a single institutional experience of 155 cases of CRS and HIPEC performed at a tertiary care cancer centre in a low \& middle-income country (LMIC).

\section{Methods:}

A multidisciplinary PSM program was initiated in 2015 at a high volume tertiary care cancer centre in north India catering largely to patients belonging to low and middle income groups. Perioperative protocols applicable to practice setting were developed and a prospective structured PSM data base was created for capturing the data. All patients undergoing CRS \& HIPEC between January 2015 to December 2020 were identified and the data was analysed for clinical spectrum, surgical details, peri-operative morbidity and mortality.

\section{Results:}

A total of 232 cases of PSM underwent CRS and HIPEC during the study period. Epithelial ovarian carcinoma (56.5\%) was the most common clinical entity treated, followed by pseudomyxoma peritonei (18.5\%), colorectal carcinoma (13.4\%) and malignant mesothelioma (5.6\%). Optimal CRS could be achieved in $94.4 \%$ of patients. Cisplatin and mitomycin were the most common drugs used for HIPEC. A total of $28.0 \%$ of patients had morbidity including deep vein thrombosis, sub-acute intestinal obstruction, sepsis, burst abdomen, lymphocele, ureteric injury, acute renal failure, entero-cutaneous fistula. The overall treatment related mortality was $3.5 \%$.

\section{Conclusions:}

Results of the current study indicate that it is feasible to establish a successful CRS and HIPEC program for PSM in LMIC facing resource constraints. The most common indication for CRS and HIPEC were carcinoma ovary followed by pseudomyxoma peritonei and colorectal carcinoma. Overall morbidity and mortality of the current series are comparable to global rates reported from high income countries. A protocol based multidisciplinary team approach, optimal patient selection and surgical expertise plays a crucial role for optimal outcomes.

\section{Background}

Peritoneal surface malignancies (PSM) comprises a group of neoplasms which either disseminate through or arise from peritoneal membrane[1]. Till recently PSM was considered as incurable advanced 
stage disease with lack of effective therapy and limited survival. The therapeutic approach to PSM was nihilistic and involved attempts at debulking surgery and delivering palliative chemotherapy. However, during last two decades significant advanced were made in the field of PSM and cytoreductive surgery (CRS) in combination with hyperthermic intraperitoneal chemotherapy (HIPEC) has emerged as a promising intervention in a subset of PSM patients [2].

Since CRS and HIPEC is a complex, multidisciplinary and resource intensive intervention, very few centers could accomplish proficiency in this type of intervention. Initial experience of CRS and HIPEC was associated with high morbidity and mortality and a significant learning curve [3]. However, increasing number of centres globally have initiated successful CRS and HIPEC programs in the recent past, largely due to standardization of patient selection criteria, surgical technique, HIPEC and peri-operative protocols. To consolidate the evidence base, a large number of prospective randomized trials are currently being conducted by many centres.

Most of the literature related to CRS and HIPEC is being published by centres from high income countries (HIC). There is paucity of literature from low and middle-income countries (LMIC) despite having a significant volume of patients with PSM. The challenges in establishing complex and expensive treatment modality like CRS and HIPEC in LMIC include lack of expertise, resource constraints, high patient volumes and socioeconomic factors.

In this article we present our experience of establishing a PSM program at a tertiary care cancer center in LMIC and present the outcomes of treatment in 232 PSM patients treated with CRS and HIPEC.

\section{Methods}

The data was collected from a prospectively maintained computerized database of department of surgical oncology based in a tertiary care comprehensive cancer centre in northern part of India. All patients who had undergone CRS and HIPEC between January 2015 and December 2020 were included in the study. Records of these patients were reviewed for details regarding the clinical spectrum, surgical details, perioperative outcomes including morbidity and mortality were analyzed.

\section{Details of Program and Protocol development:}

The team for management of peritoneal surface malignancies at our centre is multidisciplinary in nature. The team members include experienced clinicians from surgical, oncology, medical oncology, radiodiagnosis and pathology. The core surgical team comprised of experienced senior surgical oncologists, dedicated onco-anesthesia and critical care team and trained nursing and operation theatre technicians. The supportive team helps in prehabilitation and post-operative care of the patient and included members from physiotherapy, nutrition, stoma care and clinical psychology divisions. All the procedures were performed mostly by two lead senior surgical oncologists experienced in handling complex surgical oncology procedures and re-do abdominal surgeries after attending dedicated CRS and HIPEC workshops/courses and short term observerships in high volume PSM centers. 
The prehabilitation protocol included nutritional status assessment using Subjective Global Assessment (SGA) followed by intensive nutritional support in patients with average or poor nutritional status (viz. low BMI, significant weight loss, low albumin). Assessment of the baseline physical status is done by a "6minute walk test", pulmonary function test (PFT) and cardio-pulmonary exercise testing (CPET). Respiratory conditioning is done by the physiotherapy team with incentive spirometry and a program of home exercises. Cardiovascular status assessment and optimization was done by the anesthesiology and cardiology team in patients with such co-morbidities.

A standard patient selection criteria based on guidelines available was followed[4]. PSM patients with a good performance status (ECOG PS $\leq 1$ ), no or well-controlled co-morbidities were offered CRS and HIPEC and patients with unfavorable tumor biology (e.g. signet ring cell adenocarcinomas, sarcomatoid variant of malignant mesothelioma) and very high CT-PCl deemed unsuitable for optimal cytoreduction were excluded. Advanced ovarian cancer patients with high clinical tumor burden were offered neoadjuvant chemotherapy (NACT) followed by interval CRS and HIPEC and a subset of colorectal cancer patients with peritoneal metastases also received NACT. Meticulous exploratory laparotomy was performed first for calculation of $\mathrm{PCl}$ and predicting feasibility of optimal CRS. The goal of CRS was to achieve optimal cytoreduction which was defined as no macroscopic residual disease (CC-0) or macroscopic disease nodules $\leq 2.5 \mathrm{~mm}$ in size (CC-1). Larger macroscopic disease (CC-2/CC-3) were considered as sub-optimal cytoreduction[5]. Total or disease specific partial peritonectomy was performed to achieve optimal cytoreduction at the discretion of the operating surgeon [6, 7]. Multi-visceral resections were performed when indicated to achieve optimal CRS status.

After completion of cytoreductive surgery, HIPEC was instituted using a Sunchip ${ }^{T M}$ machine (manufactured by GamidaTech ${ }^{\circledR}$ France). A semi-open technique was used with the help of Omnitract ${ }^{\mathrm{TM}} / \mathrm{Th}$ mompson ${ }^{\mathrm{TM}}$ retractor and suspension of abdominal wall edges. A Steri-Drape ${ }^{\mathrm{TM}}$ was used to temporarily cover the laparotomy wound with a central opening to ensure adequate filling of perfusate and for agitation of abdominal contents for uniform distribution of heated perfusate (Fig. 1).

Chemotherapeutic agents used were Cisplatin and Mitomycin-C in majority of patients. Normal saline was used as carrier solution and HIPEC was instituted for 45 to 60 minutes at a temperature range of 41 to $42^{\circ} \mathrm{C}$. Standard safety precautions were followed including prevention of spillage, smoke evacuation and personnel protective equipment. Intraoperatively, standard protocols were followed by anesthesia team pertaining to maintenance of core temperature, hemodynamic stability, infusion of IV fluids/blood products and maintaining adequate urine output. Post-operatively, patients were monitored in ICU for 24 to 48 hours. All the morbidities related to CRS and HIPEC were documented meticulously in a predesigned proforma. After discharge patients were reviewed again by the multi-disciplinary team to assess the need for systemic therapy based on primary tumor type, surgical outcome and final histopathology report.

The cost of the CRS and HIPEC treatment was subsidized by the hospital and patients were mostly charged for disposable HIPEC sets making the treatment affordable to patients belonging to low and middle socio-economic strata. 
A descriptive analysis was performed to analyze clinical spectrum, surgical details, morbidity and mortality.

\section{Results}

A total of 232 patients with PSM underwent CRS and HIPEC between January 2015 and December 2020. The year-wise frequency of cases is shown in Fig. 2. Most of the protocols were standardized during the first year and subsequently minor modifications were added. There was a steady increase in the volume of CRS and HIPEC cases during the study period. However, only a limited number of cases were performed in the year 2020, owing to the pandemic.

The details of clinical profile of patients is shown in Table 1. The mean age was 47.3 years and there was female preponderance. Nearly one-third of patients (34.9\%) had co-morbidities, predominantly diabetes and hypertension.

Table 1

Demography and patient profile

\begin{tabular}{|lll|}
\hline Age [Mean \pm SD] & $47.3 \pm 9.2$ years & \\
\hline Sex & Male & $36(15.5 \%)$ \\
& Female & $196(84.5 \%)$ \\
\hline Performance Status (ECOG) & PS 0/1 & $214(92.2 \%)$ \\
& PS 2 & $18(7.8 \%)$ \\
& PS 3/4 & $0 \%$ \\
\hline Co-morbidities & None & $151(65.1 \%)$ \\
& Co-morbidity & $51(21.9 \%)$ \\
& $\cdot$ Diabetes mellitus & $33(14.2 \%)$ \\
& $\cdot$ Hypertension & $12(5.2 \%)$ \\
& $\cdot$ Hypothyroidism & $5(2.2 \%)$ \\
& $\cdot$ Coronary artery disease & $3(1.3 \%)$ \\
& $\cdot$ CopD & \\
\hline
\end{tabular}

Table 2 shows spectrum of PSM patients undergoing CRS and HIPEC. Ovarian carcinoma, pseudomyxoma peritonei and colorectal cancers comprised $88.4 \%$ of cases in the current series. Rare and uncommon indications comprised $11.6 \%$ of cases. 
Table 2

Clinical indications for CRS and HIPEC

\begin{tabular}{|lll|}
\hline Clinical indication & Number & Percentage \\
\hline Carcinoma Ovary & 131 & $56.5 \%$ \\
\hline Pseudomyxoma peritonei & 43 & $18.5 \%$ \\
\hline Colorectal Cancer & 31 & $13.4 \%$ \\
\hline Malignant Mesothelioma & 13 & $5.6 \%$ \\
\hline Gastric Carcinoma & 6 & $2.6 \%$ \\
\hline Endometrial Stromal Sarcoma & 5 & $2.2 \%$ \\
\hline Granulosa Cell Tumor & 3 & $1.3 \%$ \\
\hline
\end{tabular}

Table 3 shows surgical details of patients undergoing CRS and HIPEC. Mean operating time was $379 \pm 108.7$ minutes. The mean peritoneal carcinomatosis index $(\mathrm{PCl})$ was 9.3 with a range of 3 to 26 . The mean $\mathrm{PCl}$ of non-ovarian cancer group was higher than ovarian cancer group due to usage of neoadjuvant chemotherapy leading to downstaging in majority of ovarian cancer patients. Total peritonectomy was performed in $21.1 \%$ of cases and this decision was based on disease type and extent of peritoneal involvement. $94.4 \%$ of patients had an optimal CRS (CC-0/CC-1) before institution of HIPEC. Multi-visceral resection was performed in $24.5 \%$ of patients. Cisplatin was the most commonly used drug for HIPEC. The patients spent an average of 1.3 days in ICU and 8.1 days in hospital postoperatively. 
Table 3

Details of CRS and HIPEC

\begin{tabular}{|lll|}
\hline $\begin{array}{l}\text { Operative time } \\
\text { [Mean } \pm \text { SD] }\end{array}$ & $379.9 \pm 108.7$ minutes. \\
\hline PCI & Total $(\mathrm{n}=232)$ & $9.3(0-26)$ \\
{$[$ Mean (Range)] } & - Non-ovarian Group $(\mathrm{n}=101)$ & $-11.1(0-25)$ \\
& - Ovarian Group $(\mathrm{n}=131)$ & $-7.9(0-26)$ \\
\hline Extent of Peritonectomy & Total Peritonectomy & $49(21.1 \%)$ \\
\hline Types of CRS & Disease specific peritonectomy & $183(78.9 \%)$ \\
\hline Drugs for HIPEC & Optimal CRS (CC-0/CC-1) & $219(94.4 \%)$ \\
& Suboptimal CRS (CC-2/CC-3) & $13(5.6 \%)$ \\
\hline Cisplatin & $191(82.3 \%)$ \\
& Mitomycin C & $27(11.6 \%)$ \\
\hline ICU stay & Oxaliplatin & $7(3.0 \%)$ \\
\hline Post-operative Hospital stay & 8.1 (5-26) days & $2(2.2 \%)$ \\
\hline
\end{tabular}

Table 4 shows details of peri-operative morbidity (NCI-CTCAE v5.0 Gr. $3-4$ ) which occurred in $28.0 \%$ of patients. The most common major morbidity was deep venous thrombosis followed by subacute intestinal obstruction (SAIO), sepsis, burst abdomen, lymphocele, neutropenia, ureteric injury, acute renal failure and enterocutaneous fistula 
Table 4

Spectrum and incidence of peri-operative mobidity

\begin{tabular}{|lll|}
\hline Morbidity & Number of patients & Percentage \\
\hline Deep Venous Thrombosis & 15 & $6.5 \%$ \\
\hline Subacute Intestinal Obstruction & 14 & $6.0 \%$ \\
\hline Sepsis & 12 & $5.2 \%$ \\
\hline Burst Abdomen & 10 & $4.3 \%$ \\
\hline Neutropenia & 9 & $3.9 \%$ \\
\hline Lymphocele & 9 & $3.9 \%$ \\
\hline Ureteric Injury & 7 & $3.0 \%$ \\
\hline Acute Renal Failure & 4 & $1.7 \%$ \\
\hline Anastomotic Leak & 4 & $1.7 \%$ \\
\hline Pulmonary Embolism & 3 & $1.3 \%$ \\
\hline Acute Respiratory Distress Syndrome (ARDS) & 2 & $0.9 \%$ \\
\hline GTCS + Cardiac Arrest (revived) & 1 & $0.4 \%$ \\
\hline Bile Leak & 1 & $0.4 \%$ \\
\hline Overall & 65 & $28.0 \%$ \\
\hline
\end{tabular}

Peri-operative mortality occurred in 8 cases (3.5\%). 4 other patients died due to sepsis leading to acute respiratory distress syndrome (ARDS), 2 patients had sudden death on post-operative day 1 due to suspected pulmonary embolism, 1 patient succumbed to biliary peritonitis and 1 patient due to cardiac arrest in postoperative period.

\section{Discussion}

Traditionally peritoneal surface malignancies (PSM) were considered as advanced and incurable cancers and the management appraoch was generally palliative. However, during the last two decades, with the advances made in the field of CRS and HIPEC, peritoneal surface dissemination of abdominal malignancies is increasingly being recognised as a regional disease amenable to potential cure in a subset of patients. One of the major breakthroughs that helped in mainstreaming CRS \& HIPEC include surgical standardization of total peritonectomy by Paul H. Sugarbaker in 1995[6, 8]. Parallel advancements in the field of HIPEC resulted in increasing utilization of CRS \& HIPEC as an effective combination therapy for PSM by a number of centres [2]. Last decade has witnessed a spurt in publication of literature pertaining to CRS and HIPEC mainly from HIC. Due to the complexity of treatment, cost factors and associated higher morbidity and mortality very few centres from LMIC have 
ventured in to CRS and HIPEC programs despite having a significant volume of patients with PSM. There is a need to share the experience of CRS and HIPEC from resource constrained settings to assess the feasibility, safety and efficacy.

We present our experience of first 155 cases of CRS and HIPEC perfomed at a tertiary care cancer centre in India, a lower-middle income country.

CRS and HIPEC is a time and resource intensive intervention and for initiation of long term viable CRS \& HIPEC program good healthcare infrastructure and a multidisciplinary team appraoch is essential. Other important issue is optimal patient selection for CRS \& HIPEC. These procedures should only be offered to group of patients who are likely to benefit to optimize resource utilization. Two factors need to be considered while selecting patients for CRS and HIPEC. First is the disease type and second is the extent and volume of peritoneal spread. Based on current literature pseudomyxoma peritonei, mesothelioma, peritoneal metastases of tumors of appendicular and colorectal origin and ovarian cancers have shown benefit with CRS and HIPEC. Volume and extent of peritonel spread can be assessed by CT-PCI score and feasibility of optimal cytoreduction plays a crucial role in decision making for CRS and HIPEC. Performance status of the patient and presence or absence of co-morbidities is also a key factor in patient selection process.

We followed a stringent patient selection criteria in the current study.

Two thirds of the patients had no co-morbidity and the remaining had co-existing diseases like diabetes mellitus, hypertension, hythothyroidism which were well controlled. A conscious decision was also taken to exclude patients with aggressive histologies and very high tumor burden.

Spectrum of patients undergoing CRS and HIPEC varies in different studies. Factors which can influence the disease spectrum include type of surgical specialty and referral patterns. Most of the studies from the west related to CRS \& HIPEC have a preponderence of tumors of gastro-intestinal tract origin. In a study published by Levine et al comprising 1000 patients, $47.2 \%$ of the tumors were of appendiceal origin, $24.8 \%$ colorectal origin and only $6.9 \%$ were that of ovarian origin. [9-11]. In the current study more than half of the patients had tumors of ovarian origin. Peritoneal metastases of colorectal origin and pseudomyxoma peritonei comprised one fourth of the volume. These trends are likely to change over a time period due to referral patterns and revision of guidelines based on results from ongoing randomized studies.

The average peritoneal carcinomatosis index $(\mathrm{PCl})$ in our series was 9.3, lower than that reported in other contemporary series where mean $\mathrm{PCl}$ ranged from 11 to 16 [9, 12-14]. Perhaps, this is because of a stringent selection criteria used and preponderance of cases of carcinoma ovary receiving prior chemotherapy resulting in down staging and low PCI. The mean $\mathrm{PCl}$ for non-ovarian cancer patients was 11.1 in our series.

In view of the complexity and variations in protocols reported in literature, every institution planning to start CRS and HIPEC programs should have standard and uniform protocols pertaining to patient 
selection, prehabilitation, surgical technique, HIPEC methods, anesthesia and ICU care for consistent outcomes. Meticulous documentation of patient and treatment details, preferbly in a structured electronic format is important, which helps in performing audits for learning and facilitates analysis of quality data for outcomes .

As far as surgical procedures are concerned, preliminary meticulous exploration of abdomen to document $\mathrm{PCl}$ and assessment of feasibility of optimal CRS is a very critical step which will help in early decision making. We suggest a senior and experienced surgeon be involved in this part of the procedure. It is recommended to have a team of surgeons well versed with intricacies of CRS and they can work as teams to avoid fatigue and errors. At our institution two experienced surgeons performed majority of the procedures during the intial phase and susequently trainees were also allowed to perform simple peritonectomies under supervision. The main surgical goal is to achive an optimal CRS status, balancing risk versus benefit. In the current study, $21.1 \%$ had total peritonectomy and the remaining had partial disease specific peritonectomy. Approximately one fourth of patients required multi visceral resection. For the HIPEC phase of surgery, adherence to safety protocols using safe and efficacious cytotoxic agents is key to success. Dosages of prior chemotherapy a patient has received should always be kept in mind while deciding the intraperitoenal chemotherapy dose so as to avoid toxicity.

Most of the initial studies reported a very high morbidity and mortality with CRS and HIPEC $[15,16]$. However, with increasing experience contemporary studies are reporting an acceptable rate of morbidity and mortality $[10,17,18]$. In the current study, peri-operative morbidity occurred in $28.0 \%$ of patients. The most common morbidities encountered were deep vein thrombosis, subacute intestinal obstruction, sepsis and burst abdomen. Morbidity of the current study is comparable to the morbidity reported in recent studies. Overall mortality in our experience was $3.5 \%$ which was acceptable in comparison to mortalities reported in recent studies reporting a mortality rate ranging from 2 to $8.6 \%$.

\section{Conclusion}

In conclusion, outcomes of the current study indicate that it is feasible to implement complex and resource intensive treatment like CRS and HIPEC in LMIC. However, the key to success is multidisciplinary team approach, protocol based treatment delivary with strict adherence to patient selection criteria, surgical quality control and optimal peri-operative care .

\section{Abbreviations}

- CRS - Cytoreductive surgery

- HIPEC - Hyperthermic intraperitoneal chemotherapy

- PSM - Peritoneal surface malignancy

- LMIC - Low \& middle income country

- HIC - High income country 
- SGA- Subjective Global Assessment

- PFT - Pulmonary function test

- CPET - Cardio-pulmonary exercise testing

- ECOG - Eastern Cooperative Oncology Group

- PS - Performance Status

- NACT - Neo-adjuvant chemotherapy

- CC-Completeness of cytoreduction

- IV - intravenous

- ICU - Intensive care unit

- CT - Computed tomography

- $\mathrm{PCl}$ - Peritoneal carcinomatosis index

- NCI-CTCAE - National Cancer Institute - Common Terminology Criteria for Adverse Events

- Gr. - Grade

- SAIO - Sub-acute intestinal obstruction

- ARDS - Acute respiratory distress syndrome

\section{Declarations}

- Ethics approval and consent to participate - In view of the study being retrospective in nature, the AlIMS Institutional Ethics Committee waived off the requirement for ethical approval and consent.

- Consent for publication - Not applicable

- Availability of data and materials - The data was extracted from the computerized database in the Department of Surgical Oncology at AlIMS, New Delhi and will be available from corresponding author on reasonable request.

- Competing intersts - The authors declare that they have no competing interests.

- Funding - None

- Author's contributions -

- SD established the PSM program in AIIMS, New Delhi and is the lead surgeon. He provided extensive guidance in development of the manuscript.

- MR and S Bhoriwal are consultant surgeons. They reviewed the manuscript and provided valuable inputs.

- BB analyzed and interpreted the data and wrote the manuscript.

- S Bhatnagar, RG and NG were the lead anethesiologists and critical care experts. They reviewed the manuscript and provided valuable inputs.

- AS and LK were the lead medical oncologists. They reviewed the manuscript and provided valuable inputs. 
- ST and ED were the lead radiologists. They reviewed the manuscript and provided valuable inputs.

- SM and PD were the lead pathologists. They reviewed the manuscript and provided valuable inputs.

- Acknowledgements - None

- Author's information - None

- Footnotes - None

\section{References}

1. Sugarbaker PH (1999) Management of peritoneal-surface malignancy: the surgeon's role. Langenbecks Arch Surg 384:576-587. https://doi.org/10.1007/s004230050246

2. Neuwirth MG, Alexander HR, Karakousis GC (2016) Then and now: cytoreductive surgery with hyperthermic intraperitoneal chemotherapy (HIPEC), a historical perspective. J Gastrointest Oncol 7:18-28. https://doi.org/10.3978/j.issn.2078-6891.2015.106

3. Kusamura S, Baratti D, Deraco M (2012) Multidimensional analysis of the learning curve for cytoreductive surgery and hyperthermic intraperitoneal chemotherapy in peritoneal surface malignancies. Ann Surg 255:348-356. https://doi.org/10.1097/SLA.0b013e3182436c28

4. International PSOGI-recommendations for the management of peritoneal metastases. In: PSOGI. http://www.psogi.com/psogi/international-recommendations-for-the-management-of-peritonealmetastases/. Accessed 30 Apr 2020

5. Sugarbaker PH (1996) Peritoneal Carcinomatosis: Principles of Management. Springer Science \& Business Media

6. Sugarbaker PH (1995) Peritonectomy procedures. Ann Surg 221:29-42

7. Jacquet P, Sugarbaker PH (1996) Current methodologies for clinical assessment of patients with peritoneal carcinomatosis. J Exp Clin CANCER Res 15:49-58

8. Wimberger P, Wehling M, Lehmann N, et al (2010) Influence of residual tumor on outcome in ovarian cancer patients with FIGO stage IV disease: an exploratory analysis of the AGO-OVAR (Arbeitsgemeinschaft Gynaekologische Onkologie Ovarian Cancer Study Group). Ann Surg Oncol 17:1642-1648. https://doi.org/10.1245/s10434-010-0964-9

9. Levine EA, Stewart JH, Shen P, et al (2014) Cytoreductive Surgery and Hyperthermic Intraperitoneal Chemotherapy for Peritoneal Surface Malignancy: Experience with 1,000 Patients. J Am Coll Surg 218:573-585. https://doi.org/10.1016/j.jamcollsurg.2013.12.013

10. Piso P, Nedelcut SD, Rau B, et al (2019) Morbidity and Mortality Following Cytoreductive Surgery and Hyperthermic Intraperitoneal Chemotherapy: Data from the DGAV StuDoQ Registry with 2149 Consecutive Patients. Ann Surg Oncol 26:148-154. https://doi.org/10.1245/s10434-018-6992-6

11. Moran B, Cecil T, Chandrakumaran K, et al (2015) The results of cytoreductive surgery and hyperthermic intraperitoneal chemotherapy in 1200 patients with peritoneal malignancy. Colorectal 
Dis 17:772-778. https://doi.org/10.1111/codi.12975

12. Cascales Campos P, Gil J, Parrilla P (2014) Morbidity and mortality outcomes of cytoreductive surgery and hyperthermic intraperitoneal chemotherapy in patients with primary and recurrent advanced ovarian cancer. Eur J Surg Oncol J Eur Soc Surg Oncol Br Assoc Surg Oncol 40:970-975. https://doi.org/10.1016/j.ejso.2013.08.013

13. Glehen O, Gilly FN, Boutitie F, et al (2010) Toward curative treatment of peritoneal carcinomatosis from nonovarian origin by cytoreductive surgery combined with perioperative intraperitoneal chemotherapy: a multi-institutional study of 1,290 patients. Cancer 116:5608-5618. https://doi.org/10.1002/cncr.25356

14. Tabrizian P, Jibara G, Shrager B, et al (2013) Outcomes for cytoreductive surgery and hyperthermic intraperitoneal chemotherapy in the elderly. Surg Oncol 22:184-189. https://doi.org/10.1016/j.suronc.2013.06.001

15. Elias D, Goere D, Blot F, et al (2007) Optimization of Hyperthermic Intraperitoneal Chemotherapy With Oxaliplatin Plus Irinotecan at $43^{\circ} \mathrm{C}$ After Compete Cytoreductive Surgery: Mortality and Morbidity in 106 Consecutive Patients. Ann Surg Oncol 14:1818-1824. https://doi.org/10.1245/s10434-0079348-1

16. Deraco M, Baratti D, Kusamura S (2007) Morbidity and Quality of Life following Cytoreduction and HIPEC. Perit Carcinomatosis 403-418. https://doi.org/10.1007/978-0-387-48993-3_26

17. Eveno C, Schiavone R, Pocard M, et al (2017) Effect of postoperative morbidity on survival after cytoreductive surgery (CRS) with heated intraperitoneal chemotherapy (HIPEC) for peritoneal metastasis in a series of 700 cases. J Clin Oncol 35:3565-3565. https://doi.org/10.1200/JC0.2017.35.15_suppl.3565

18. Votanopoulos KI, Newman NA, Russell G, et al (2013) Outcomes of Cytoreductive Surgery (CRS) with Hyperthermic Intraperitoneal Chemotherapy (HIPEC) in Patients Older Than 70 Years; Survival Benefit at Considerable Morbidity and Mortality. Ann Surg Oncol 20:3497-3503.

https://doi.org/10.1245/s10434-013-3053-z

\section{Figures}



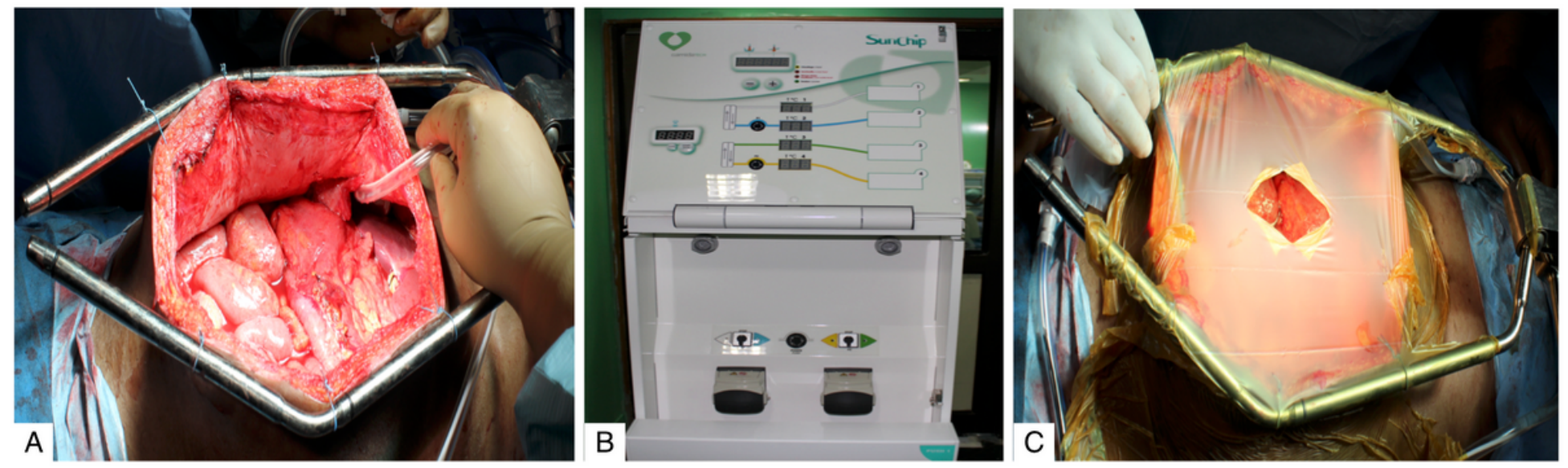

\section{Figure 1}

HIPEC Technique. (A) Suspension of abdominal wall edges to OmnitractTM retractor to create a Coliseum (B) SunchipTM machine (C) Coliseum covered with Steri-DrapeTM (semi-open). 


\section{Year-wise Frequency}

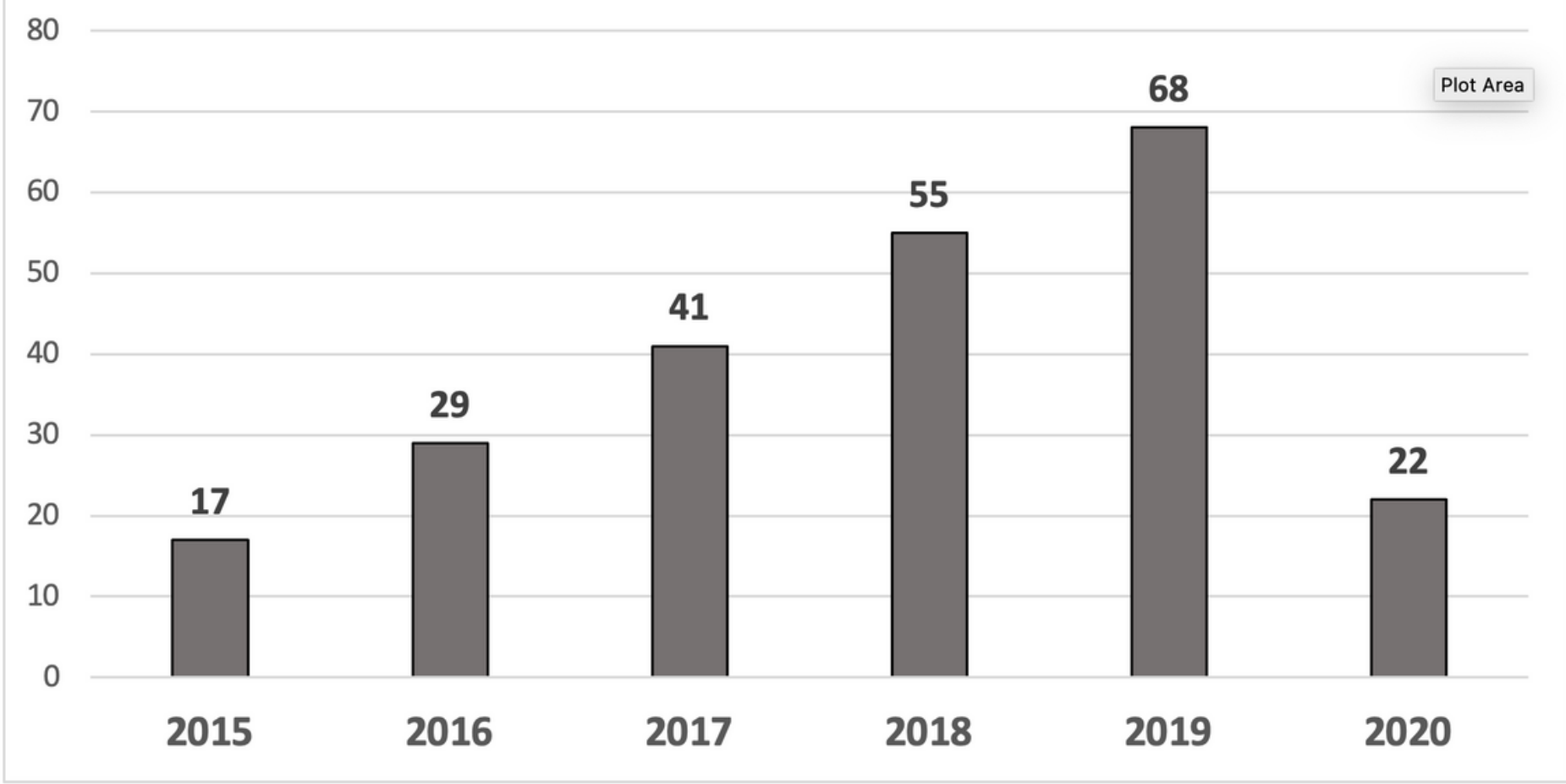

Figure 2

Year-wise trends of CRS \& HIPEC 